\title{
Probabilistic Sophistication and Variational Preferences
}

\section{Citation}

Strzalecki, Tomasz. 2011. "Probabilistic sophistication and variational preferences." Journal of Economic Theory 146 (5) (September): 2117-2125.

\section{Published Version}

doi:10.1016/j.jet.2011.06.010

\section{Permanent link}

http://nrs.harvard.edu/urn-3:HUL.InstRepos:11352635

\section{Terms of Use}

This article was downloaded from Harvard University's DASH repository, and is made available under the terms and conditions applicable to Open Access Policy Articles, as set forth at http:// nrs.harvard.edu/urn-3:HUL.InstRepos:dash.current.terms-of-use\#OAP

\section{Share Your Story}

The Harvard community has made this article openly available.

Please share how this access benefits you. Submit a story.

\section{Accessibility}




\title{
Probabilistic Sophistication and Variational Preferences*
}

\author{
Tomasz Strzalecki ${ }^{\dagger}$ \\ Harvard University
}

\begin{abstract}
This paper shows that in the class of variational preferences the notion of probabilistic sophistication is equivalent to expected utility as long as there exists at least one event such that the independence axiom holds for bets on that event. This extends a result of Marinacci [13] and provides a novel interpretation of his result.

Keywords:

ambiguity aversion, probabilistic sophistication, expected utility, variational preferences.

JEL Codes: D0, D8
\end{abstract}

*This note is a revised and extended version of Chapter 9 of my dissertation at Northwestern University; some of the results were also reported in my job market paper. Part of this research was done while I was visiting the Economic Theory Center at Princeton University, to which I'm very grateful for its support and hospitality. I would like to thank Eddie Dekel, Peter Klibanoff, Massimo Marinacci, Jan Werner, as well as an associate editor and a referee for their very helpful comments and suggestions. All errors are my own. This version: May 15, 2011.

${ }^{\dagger}$ Dept. of Economics, Harvard University, Littauer Center, 1805 Cambridge Street, Cambridge, MA 02138, USA. E-mail: tomasz_strzalecki@harvard.edu 


\section{Introduction}

This paper studies two well known classes of preferences: the variational preferences of Maccheroni, Marinacci, and Rustichini [11] and the probabilistically sophisticated preferences of Machina and Schmeidler [12].

Variational preferences are a very broad class of preferences that allow for modeling choices consistent with the Ellsberg [5] paradox. This class of preferences includes the maxmin expected utility (MEU) preferences of Gilboa and Schmeidler [6], where the decision maker has a set of priors, rather than a unique probability, as well as many other classes of preferences that violate separability across states.

The notion of probabilistic sophistication means that the decision maker bases his choices on probabilistic beliefs. This class includes expected utility, as well as many nonexpected utility criteria that allow for modeling the Allais [1] paradox and related violations of linearity in probabilities.

In many situations involving ambiguity and ambiguity aversion, such as in the Ellsberg paradox, there exist events to which the decision maker attaches unambiguous probabilities. In principle, a decision maker could be probabilistically sophisticated but nonexpected utility over such events. The question that this paper studies is to what extent it is possible to model such attitude using variational preferences.

Marinacci [13] studied this question for the MEU preferences and showed that under an assumption that all the probabilities in the set of priors agree on some event, probabilistic sophistication is equivalent to expected utility. The contribution of this paper is twofold. First it characterizes Marinacci's assumption axiomatically: it shows that it is equivalent to the existence of an event $A$ such that the independence axiom holds for bets on $A$. In light of this theorem, Marinacci's result can be interpreted as pointing to the inability of MEU preferences to accommodate expected utility and nonexpected utility within the same model. The second contribution of this paper is an extension (using different proof techniques) of Marinacci's result to the class 
of variational preferences: they too are unable to accommodate expected utility and nonexpected utility within the same model.

\section{Preliminaries}

\subsection{Setting}

Let $S$ be the set of states of the world with a sigma algebra $\Sigma$ of subsets of $S$. Let $\Delta(S)$ denote the set of all finitely additive probability distributions on $(S, \Sigma)$ and let $\Delta^{\sigma}(S)$ denote the set of all countably additive probabilities. Let $X$ be the set of consequences, assumed to be a convex subset of a vector space. An act is a $\Sigma$-measurable and finite-valued mapping $f: S \rightarrow X$ that attaches a consequence to each possible state. Let $\mathcal{F}$ denote the set of all acts. The preferences $\succsim$ are defined on $\mathcal{F}$. If $f, g \in \mathcal{F}$ and $A \subseteq S$, then $f_{A} g$ denotes an act with $f_{A} g(s)=f(s)$ if $s \in A$ and $f_{A} g(s)=g(s)$ if $s \notin A$. For any algebra of events $\mathcal{A} \subseteq \Sigma$ let $\mathcal{F}_{\mathcal{A}}$ denote the set of acts in $\mathcal{F}$ that are measurable with respect to $\mathcal{A}$. In particular, if $\mathcal{A}=\left\{\emptyset, A, A^{c}, S\right\}$ for some $A \in \Sigma$, then $\mathcal{F}_{\mathcal{A}}$ is denoted simply by $\mathcal{F}_{A}$.

\subsection{Probabilistic sophistication}

The notion of probabilistic sophistication means that the decision maker treats subjective uncertainty as if it was objective risk; he cares only about the probabilities of events, not the events themselves. ${ }^{1}$ Such decision maker formulates a subjective probability measure on the state space. Any two acts that imply the same distribution over outcomes are being treated in the same way. More formally, a preference is probabilistically sophisticated if there exists a nonatomic probability measure $q \in \Delta(S)$ such that for any two acts $f$

\footnotetext{
${ }^{1}$ This notion was introduced by Machina and Schmeidler [12] and subsequently extended by Grant [7] and Chew and Sagi [4]; the latter approach is being adopted here.
} 
and $g$

$$
q(s \in S \mid f(s)=x)=q(s \in S \mid g(s)=x) \text { for all } x \in X \Rightarrow f \sim g
$$

\subsection{Variational Preferences}

The variational preferences, introduced and axiomatized by Maccheroni et al. [11], are represented by

$$
V(f)=\min _{p \in \Delta S} \int_{S} u(f) \mathrm{d} p+c(p)
$$

where the function $c: \Delta(S) \rightarrow[0, \infty]$ is convex, weak* lower semicontinuous, and grounded (takes value zero for some $p \in \Delta(S)$ ); and $u: X \rightarrow \mathbb{R}$ is a nonconstant and affine utility function. For the purpose of this paper it will be also assumed that $u$ is unbounded. An important subclass of variational preferences are those where the minimization is over the set of countably additive probabilities. Such preferences are called continuous variational preferences.

A classic example of variational preferences are maxmin expected utility preferences of Gilboa and Schmeidler [6] with representation

$$
V(f)=\min _{p \in P} \int_{S} u(f) \mathrm{d} p,
$$

where $P$ is a nonempty, convex, and weak* compact set of probabilities in $\Delta(S)$. Formula $(2)$ is a special case of $(1)$ for

$$
c_{\mathrm{MEU}}(p)= \begin{cases}0 & \text { for } p \in P \\ \infty & \text { for } p \notin P .\end{cases}
$$

A special case of both of those classes are Anscombe-Aumann expected util- 
ity preferences represented by

$$
V(f)=\int_{S} u(f) \mathrm{d} p
$$

in this case the set $P$ is a singleton composed of $p$.

\section{Results}

\subsection{Binary Independence}

Definition 1. (Nontrivial event) An event $A \in \Sigma$ is nontrivial if and only if there exists $x, y \in X$ with $x \succ x_{A} y \succ y$.

Axiom A1 postulates the existence of a nontrivial event $A$ such that the independence axiom holds for bets on $A$.

Axiom A1 (Binary Independence). There exists a nontrivial event $A \in \Sigma$ such that

$$
f \sim g \Rightarrow \frac{1}{2} f+\frac{1}{2} h \sim \frac{1}{2} g+\frac{1}{2} h \quad \text { for all } f, g, h \in \mathcal{F}_{A}
$$

Recall that acts in $\mathcal{F}_{A}$ have the form $x A y$, that is, they are bets based on $A$. More generally, an algebra $\mathcal{A} \subseteq \Sigma$ is nontrivial if and only if it contains a nontrivial event. For example, if $A$ is a nontrivial event, then the algebra $\left\{\emptyset, A, A^{c}, S\right\}$ is nontrivial. It is easily seen that the Binary Independence axiom can be equivalently formulated in terms of the existence of a nontrivial subagebra $\mathcal{A}$ of $\Sigma$ such that independence holds on $\mathcal{F}_{\mathcal{A}}$.

\subsection{Maxmin Expected Utility}

The main result of this paper determines the extent to which the variational preferences can be used for modeling the Allais paradox. Marinacci [13] showed that for the subclass of maxmin expected utility preferences this 
extent is limited. They collapse to expected utility preferences under an assumption of agreement of probabilities

Assumption 1. There exists an event $A \in \Sigma$ such $0<p(A)=p^{\prime}(A)<1$ for all $p, p^{\prime} \in P$.

This assumption means that there exists an event $A$ such that any two measures belonging to the set of priors $P$ agree on $A$. This assumption is expressed in terms of the representation; the following result provides a behavioral characterization of Assumption 1.

Theorem 1. Suppose that $\succsim$ is a maxmin expected utility preference. Assumption 1 holds if and only if Axiom A1 holds.

The main result of this paper, Theorem 3 below, extends the result of Marinacci [13] to the whole class of variational preferences under an appropriately extended notion of agreement of probabilities.

\subsection{Variational Preferences}

In principle, there are two possible extensions of this assumption to cost functions taking values other than zero and infinity, i.e., to the whole class of variational preferences. They both reduce to the assumption of Marinacci [13] for the subclass of maxmin expected utility preferences.

Assumption 2. There exists an event $A \in \Sigma$ such that if $c(p), c\left(p^{\prime}\right)<\infty$, then $0<p(A)=p^{\prime}(A)<1$.

This assumption means that there exists an event $A$ such that any two measures that the decision maker considers to be at all plausible (i.e., having finite cost) attach the same probability to $A$. Similarly to maxmin expected utility preferences, Axiom A1 provides a characterization of this assumption.

Theorem 2. Suppose that $\succsim$ is an unbounded variational preference. Assumption 2 holds if and only if Axiom A1 is satisfied. 
Assumption 2 is relatively strong, especially in cases where the set of plausible measures is large. A further generalization of Assumption 1 is the following.

Assumption 3. For any $r \geq 0$ there exists an event $A_{r} \in \Sigma$ such that if $c(p)=c\left(p^{\prime}\right)=r$, then $0<p\left(A_{r}\right)=p^{\prime}\left(A_{r}\right)<1$.

This assumption requires that any two measures with the same cost agree on some event. This assumption is weaker than Assumption 2 because the event on which the measures have to agree can vary with the cost level. This requirement is more in the spirit of variational preferences where the decision maker considers various classes of probability distributions, each with a different cost. The agreement event can be different for each such class, rather than being uniform. This assumption is easier to verify given the specific functional from of $c$, which may be helpful in some cases, for example in applications, where a subclass of variational preferences is being studied. $^{2}$

The main result of this paper, Theorem 3 shows that even the weaker Assumption 3 is sufficient. The proof does not follow from Marinacci [13], but uses different techniques: it builds on the elegant characterization of probabilistically sophisticated variational preferences obtained by Maccheroni et al. $[11] .^{3}$

Theorem 3. Suppose that $\succsim$ is a continuous and unbounded variational preference. The following three statements are equivalent

(i) $\succsim$ is probabilistically sophisticated and Assumption 3 holds

(ii) $\succsim$ is probabilistically sophisticated and Axiom A1 holds

\footnotetext{
${ }^{2}$ The axiomatic characterization of Assumption 3 within the class of general variational preferences is an open question. For probabilistically sophisticated variational preferences, however, Theorem 3 establishes the equivalence between Axiom A1 and Assumption 3.

${ }^{3}$ I would like to thank Simone Cerreia-Vioglio for pointing out that an alternative proof could be obtained using a rearrangement invariance approach of Luxemburg [10]. The proof presented in this paper is elementary and does not rely on those results.
} 
(iii) $\succsim$ is an Anscombe-Aumann expected utility preference.

Remark 1. Strictly speaking, Theorem 3 is not a generalization of the result of Marinacci [13]; his theorem holds also for $\alpha$-MEU preferences, which - not being uncertainty averse - do not belong to the class of variational preferences. Moreover, his results for maxmin expected utility preferences do not rely on countable additivity.

Remark 2. Marinacci [13] shows that Assumption 1 cannot be weakened. His Proposition 2 exhibits an example of MEU preferences that are probabilistically sophisticated, yet not expected utility. Similarly, rank-dependent expected utility preferences with a concave probability transformation function, see, e.g., Quiggin [14], Yaari [17], as well as many other probabilistically sophisticated MEU preferences have this property. ${ }^{4}$ The existence of such preferences is possible because they do not satisfy Axiom A1 (there are no nontrivial events on which those preferences satisfy the independence axiom). Such examples are inherited by Theorem 3; the class of such examples is even larger, as it includes some variational but non-MEU preferences, notably the multiplier preferences of Hansen and Sargent [9].

Remark 3. In a recent paper Cerreia-Vioglio, Maccheroni, Marinacci, and Montrucchio [3] independently obtain similar results for the general class of uncertainty averse preferences characterized by Cerreia-Vioglio, Maccheroni, Marinacci, and Montrucchio [2]. They work in terms of the analog of Assumption 2 of this paper and do not consider weaker conditions like Assumption 3 in this paper. They also provide an axiomatic characterization of the analog of Assumption 2. However, their characterization is different than the one obtained in this paper: they first derive an auxiliary incomplete ordering $\succsim^{*}$

\footnotetext{
${ }^{4}$ Grant and Kajii [8] characterize the class of probabilistically sophisticated MEU preferences.

${ }^{5}$ Multiplier preferences, and more broadly divergence preferences with respect to a nonatomic $q \in \Delta^{\sigma}(S)$, are probabilistically sophisticated, but not expected utility, see Maccheroni et al. [11].
} 
(the revealed unambiguous preference) and then uncover the unambiguous event from that ordering. By contrast, Axiom A1 uncovers the unambiguous event directly from the observable preference $\succsim$.

\section{Appendix: Proofs}

Let $B_{0}(\Sigma)$ denote the set of all real-valued $\Sigma$-measurable simple functions and let $B_{0}(\Sigma, K)$ be the set of all functions in $B_{0}(\Sigma)$ that take values in a convex set $K \subseteq \mathbb{R}$.

\section{A Proof of Theorem 2}

Verifying that Assumption 2 implies Axiom A1 is routine. Turn to sufficiency and define $\Delta^{2}:=\left\{\pi \in \mathbb{R}^{2} \mid \pi_{1} \geq 0, \pi_{2} \geq 0\right.$, and $\left.\pi_{1}+\pi_{2}=1\right\}$. For any $P \subseteq \Delta^{2}$ let $\delta_{P}: \Delta^{2} \rightarrow[0, \infty]$ be defined as $\delta_{P}(\pi)=0$ if $\pi \in P$ and $\infty$ otherwise. Since $\mathcal{A}$ is notrivial, there exists a nontrivial $A \in \mathcal{A}$. Let $\mathcal{F}_{A}$ denote the set of acts measurable with respect to $\left\{\emptyset, A, A^{c}, S\right\}$.

Step 1: The restriction of $\succsim$ to $\mathcal{F}_{A}$ satisfies Order, Mixture Continuity, Monotonicity, and Independence, hence by the Anscombe-Aumann representation theorem, it has a representation $f \mapsto \pi_{1} v(f(A))+\pi_{2} v\left(f\left(A^{c}\right)\right)$ for all $f \in \mathcal{F}_{A}$ where $v: X \rightarrow \mathbb{R}$ is an affine function and $\pi \in \Delta^{2}$. Since constant acts belong to $\mathcal{F}_{A}$, the functions $u$ and $v$ are identical up to a positive affine transformation; choose $v$ to coincide with $u$. Hence, the preference on the set $B_{A}:=\left\{\left(a_{A} b\right) \in B_{0}(\Sigma, u(X)) \mid a, b \in \mathbb{R}\right\}$ induced by $\succsim$ is represented by $\left(a_{A} b\right) \mapsto \pi_{1} a+\pi_{2} b$. There is a natural isomorphism between the set $B_{A}$ and the set $(u(X))^{2}$. Let $\succsim^{2}$ denote the induced preferences on $(u(X))^{2}$. By the above, the preference $\succsim^{2}$ has a representation $\bar{I}(a, b)=a \pi_{1}+b \pi_{2}$.

Step 2: Define the function $\Pi: \Delta(S) \rightarrow \Delta^{2}$ by $\Pi(p)=(p(A), 1-p(A))$ and observe that $\Pi$ is continuous because if a net $p_{\alpha} \rightarrow^{w^{*}} p$, then in particular $p_{\alpha}(A) \rightarrow p(A)$, so $\left(p_{\alpha}(A), 1-p_{\alpha}(A)\right) \rightarrow(p(A), 1-p(A))$; hence, $\Pi\left(p_{\alpha}\right) \rightarrow$ $\Pi(p)$. Observe that for any $\pi \in \Delta^{2}$ the set $\Pi^{-1}(\pi)$ is a nonempty, convex, and 
weak* closed subset of $\Delta(S)$, hence it is weak* compact. Define the function $\hat{c}: \Delta^{2} \rightarrow[0, \infty]$ by $\hat{c}(\pi):=\min _{p \in \Pi^{-1}(\pi)} c(p)$. Clearly, $\hat{c}$ is grounded, since $c$ is. Also, it is convex: fix any $\pi, \pi^{\prime} \in \Delta^{2}$ and any $\alpha \in[0,1]$. Let $p \in \Pi^{-1}(\pi)$ and $p^{\prime} \in \Pi^{-1}\left(\pi^{\prime}\right)$ be such that $\hat{c}(\pi)=c(p)$ and $\hat{c}\left(\pi^{\prime}\right)=c\left(p^{\prime}\right)$. From convexity of $c$ it follows that $c\left(\alpha p+(1-\alpha) p^{\prime}\right) \leq \alpha c(p)+(1-\alpha) c\left(p^{\prime}\right) \leq k$. On the other hand, $\Pi\left(\alpha p+(1-\alpha) p^{\prime}\right)=\alpha \pi+(1-\alpha) \pi^{\prime}$, so $\hat{c}\left(\alpha \pi+(1-\alpha) \pi^{\prime}\right) \leq$ $c\left(\alpha p+(1-\alpha) p^{\prime}\right)$. The function $\hat{c}$ is also lower semicontinuous; to see that it is necessary to prove that the set $\hat{C}_{k}:=\left\{\pi \in \Delta^{2} \mid \hat{c}(\pi) \leq k\right\}$ is closed for any $k \in[0, \infty]$. Since the function $c$ is weak* lower semicontinuous, the set $C_{k}:=\{p \in \Delta(S) \mid c(p) \leq k\}$ is weak* closed and therefore weak* compact for any $k \in[0, \infty]$. Since continuous functions carry compact sets to compact sets the set $\Pi\left(C_{k}\right)$ is compact for any $k \in[0, \infty]$ and therefore closed Finally, observe that $\Pi\left(C_{k}\right)=\hat{C}_{k}$ for all $k \in[0, \infty]$. For any $\pi \in \Pi\left(C_{k}\right)$ there exists $p \in C_{k}$ such that $\Pi(p)=\pi$. By definition, this means that $c(p) \leq k$ and $p \in \Pi^{-1}(\pi)$. Hence, $\hat{c}(\pi) \leq c(p) \leq k$. Conversely, for any $\pi \in \hat{C}_{k}$, by definition $\hat{c}(\pi) \leq k$. Choose $p \in \Pi^{-1}(\pi)$ such that $\hat{c}(\pi)=c(p)$. This means that $c(p) \leq k$, i.e., $p \in C_{k}$, and $\Pi(p)=\pi$; it follows that $\pi \in \Pi\left(C_{k}\right)$. This establishes the weak* lower semicontinuity of $\hat{c}$. Together with convexity and groundedness establishes that $\hat{c}$ is a cost function in the sense of Maccheroni et al. [11].

Step 3: Define $I: B_{0}(\Sigma, u(X)) \rightarrow \mathbb{R}$ by $I(\xi):=\min _{p \in \Delta(S)} \int \xi \mathrm{d} p+c(p)$. Define $\hat{I}:(u(X))^{2} \rightarrow \mathbb{R}$ by $\hat{I}(a, b):=I\left(a_{A} b\right)$. Hence, $\hat{I}$ represents $\succsim^{2}$. Observe that $\hat{I}(a, b)=\min _{p \in \Delta(S)} a p(A)+b p\left(A^{c}\right)+c(p)=\min _{\pi \in \Delta^{2}} \min _{p \in \Pi^{-1}(\pi)} a \pi_{1}+$ $b \pi_{2}+c(p)=\min _{\pi \in \Delta^{2}} a \pi_{1}+b \pi_{2}+\hat{c}(\pi)$. Thus, $\bar{I}$ and $\hat{I}$ there are two variational representations of $\succsim^{2}$. By Proposition 6 of Maccheroni et al. [11], $\hat{c}=\delta_{\{\pi\}}$ for some $\pi \in \Delta^{2}$. Suppose there exist $p, q \in \Delta(S)$ such that $c(p), c(q)<\infty$ and $p(A) \neq q(A)$. Define $\pi^{p}:=\Pi(p)$ and $\pi^{q}:=\Pi(q)$ and observe that $\hat{c}\left(\pi^{p}\right)=\min _{p^{\prime} \in \Pi^{-1}\left(\pi^{p}\right)} c\left(p^{\prime}\right) \leq c(p)<\infty$ and $\hat{c}\left(\pi^{q}\right)=\min _{p^{\prime} \in \Pi^{-1}\left(\pi^{q}\right)} c\left(p^{\prime}\right) \leq$ $c(q)<\infty$. Contradiction with $\pi^{p} \neq \pi^{q}$ and $\hat{c}=\delta_{\{\pi\}}$ for some $\pi \in \Delta^{2}$. Thus, $p(A)=q(A)$ for all $p, q \in \Delta(S)$ such that $c(p), c(q)<\infty$. The nontriviality 
of $A$ implies that this common value is strictly between zero and one.

\section{B Proof of Theorem 1}

Let $I: B_{0}(S, u(X)) \rightarrow \mathbb{R}$ be equal to $I(\xi)=\min _{p \in P} \int \xi \mathrm{d} p$ for some nonempty convex and weak* compact subset of $\Delta(S)$. Proceed with Steps 1 and 2 as in the proof of Theorem 2. Observe that $\hat{c}$ obtained in Step 2 is equal to $\delta_{P^{2}}$ for some nonempty, compact and convex set $P^{2} \subseteq \Delta^{2}$. Proceed with Step 3 as in the proof of Theorem 2 except that instead of relying on Proposition 6 of Maccheroni et al. [11], rely instead on Theorem 1 (b) of Gilboa and Schmeidler [6] to conclude that $P^{2}=\{\pi\}$.

\section{Proof of Theorem 3}

Let $S$ be a set and let $\Sigma$ be a sigma algebra of its events. Let $\Delta^{\sigma}(S)$ denote the set of all countably additive probability measures on $(S, \Sigma)$. Let $q \in \Delta^{\sigma}(S)$ and let $L^{1}(S, \Sigma, q)$ denote the set of all nonnegative $\Sigma$-measurable functions on $S$ with $\int_{S} f \mathrm{~d} q=1$. For $f, g \in L^{1}(S, \Sigma, q)$ define $f \sim_{c x} g$ iff

$$
q(s \in S \mid f(s) \leq t)=q(s \in S \mid g(s) \leq t)
$$

for any $t \geq 0$. Similarly, for any measures $p, p^{\prime} \in \Delta^{\sigma}(S)$ define $p \sim_{c x} p^{\prime}$ iff

$\frac{\mathrm{d} p}{\mathrm{~d} q} \sim_{c x} \frac{\mathrm{d} p^{\prime}}{\mathrm{d} q}$. For $p \in \Delta^{\sigma}(S)$, the set $O(p)=\left\{p^{\prime} \in \Delta^{\sigma}(S, \Sigma) \mid p^{\prime} \sim_{c x} p\right\}$ is called the orbit of $p$. A set of measures $\Gamma \subseteq \Delta^{\sigma}(q)$ is called orbit-closed iff $p \in \Gamma \Rightarrow O(p) \subseteq \Gamma$.

Lemma 1. Let $f \in L^{1}(S, \Sigma, q)$ and let $F, G \in \Sigma$ be disjoint events, with $q(F)=q(G)$. Then, there exists $g \in L^{1}(S, \Sigma, q)$ such that $f=g$ on $(F \cup G)^{c}$, $\int_{F} f \mathrm{~d} q=\int_{G} g \mathrm{~d} q$, and $f \sim_{c x} g$. 
Proof. For each $n \in \mathbb{N}$ and for $1 \leq k \leq n 2^{n}$ define sets

$$
\begin{aligned}
& { }_{n} F_{0}=\{s \in F \mid f(s) \geq n\},{ }_{n} F_{k}=\left\{s \in F \mid \frac{k-1}{2^{n}} \leq f(s) \leq \frac{k}{2^{n}}\right\}, \\
& { }_{n} G_{0}=\{s \in G \mid f(s) \geq n\},{ }_{n} G_{k}=\left\{s \in G \mid \frac{k-1}{2^{n}} \leq f(s) \leq \frac{k}{2^{n}}\right\} .
\end{aligned}
$$

Because $q$ is nonatomic, it is also convex-ranged, see, e.g., Villegas [16]. Thus, for each $n$, partitions $\left\{{ }_{n} F^{\prime}{ }_{k}\right\}_{k=0}^{n 2^{n}}$ of $F$ and $\left\{{ }_{n} G^{\prime}{ }_{k}\right\}_{k=0}^{n 2^{n}}$ of $G$ can be constructed such that

$$
q\left(F_{n, k}^{\prime}\right)=q\left(G_{n, k}\right) \text { and } q\left(G_{n, k}^{\prime}\right)=q\left(F_{n, k}\right)
$$

for all $0 \leq k \leq n 2^{n}$ and

$$
{ }_{(n+1)} G_{(2 k)}^{\prime} \subseteq{ }_{(n+1)} G_{(k)}^{\prime} \text { and }{ }_{(n+1)} G_{(2 k+1)}^{\prime} \subseteq{ }_{(n+1)} G_{(k)}^{\prime}
$$

for all $0 \leq k \leq n 2^{n}$ and $n \in \mathbb{N}$.

Define functions

$$
\begin{aligned}
f_{n} & =\sum_{k=1}^{n 2^{n}}\left(\frac{k-1}{2^{n}} \mathbf{1}_{n F_{k}}\right)+n \mathbf{1}_{n F_{k}}+f_{\left.\right|_{(E \cup G)^{c}}}+\sum_{k=1}^{n 2^{n}}\left(\frac{k-1}{2^{n}} \mathbf{1}_{n} G_{k}\right)+n \mathbf{1}_{n} G_{k}, \\
g_{n} & =\sum_{k=1}^{n 2^{n}}\left(\frac{k-1}{2^{n}} \mathbf{1}_{n F^{\prime}{ }_{k}}\right)+n \mathbf{1}_{n F^{\prime}{ }_{k}}+f_{\left.\right|_{(E \cup G)^{c}}}+\sum_{k=1}^{n 2^{n}}\left(\frac{k-1}{2^{n}} \mathbf{1}_{n} G^{\prime}{ }_{k}\right)+n \mathbf{1}_{n} G^{\prime}{ }_{k} .
\end{aligned}
$$

Observe, that functions $f_{n}$ satisfy $0 \leq f_{n} \leq f_{n+1}$, and converge pointwise to $f$. Similarly, functions $g_{n}$ satisfy $0 \leq g_{n} \leq g_{n+1}$. Define $g=\lim _{n \rightarrow \infty} g_{n}$. Observe that $f=g$ on $(E \cup G)^{c}$. Moreover, $\int_{S} f_{n} \mathrm{~d} q=\int_{S} g_{n} \mathrm{~d} q$, so by the Monotone Convergence Theorem $\int_{S} f \mathrm{~d} q=\int_{S} g \mathrm{~d} q$. 
To see that $f \sim_{c x} g$, let $t \geq 0$ and define sets

$$
\begin{aligned}
& A_{n}=\left\{s \in S \mid f_{n}(s) \leq t\right\}, \quad A=\{s \in S \mid f(s) \leq t\}, \\
& B_{n}=\left\{s \in S \mid g_{n}(s) \leq t\right\}, \quad B=\{s \in S \mid g(s) \leq t\},
\end{aligned}
$$

Verify, that by construction of $f_{n}$ and $g_{n} A_{n} \downarrow A, B_{n} \downarrow B$, and $q\left(A_{n}\right)=$ $q\left(B_{n}\right)$ for all $n$. By countable additivity of $q, \lim _{n \rightarrow \infty} q\left(A_{n}\right)=q(A)$ and $\lim _{n \rightarrow \infty} q\left(B_{n}\right)=q(B)$.

Lemma 2. Suppose that $\Gamma \subseteq \Delta^{\sigma}(q)$ is an orbit-closed set of measures. Suppose also that there exists $A \in \Sigma$ such that $0<p(A)=p^{\prime}(A)<1$ for all $p, p^{\prime} \in \Gamma$. Then $\Gamma=\{q\}$.

Proof. Let $\alpha=q(A)$. Observe, that wlog $\alpha \leq \frac{1}{2}$, because if all measures in $\Gamma$ agree on $A$, then they also agree on $A^{c}$. Also, if $\alpha=0$, then for any $p \in \Gamma$ $q(A)=0 \Rightarrow p(A)=0$, contradicting the assumption. Thus, $\alpha \in\left(0, \frac{1}{2}\right]$.

Step 1: $p(E)=p(A)$ for all $p \in \Gamma$ and for all events $E \in \Sigma$ with $q(E)=\alpha$.

Let $E \in \Sigma$ be such that $q(E)=\alpha$ and observe that $q(A-E)=q(E-$ $A)$. Let $p \in \Gamma$ and define $f=\frac{\mathrm{d} p}{\mathrm{~d} q}$. By Lemma 1 applied to $(E-A)$ and $(A-E)$, there exists $g \in L^{1}(S, \Sigma, q)$ such that $f=g$ on $(A \cup E)^{c} \cup(A \cap E)$, $\int_{(E-A)} f \mathrm{~d} q=\int_{(A-E)} g \mathrm{~d} q$, and $f \sim_{c x} g$. Define measure $p^{\prime} \in \Delta^{\sigma}(S, \Sigma)$ by $p^{\prime}(F)=\int_{F} g \mathrm{~d} q$ and observe that $p^{\prime} \sim_{c x} p$. Moreover, $p(E-A)=p^{\prime}(A-E)$ and $p(A \cap E)=p^{\prime}(A \cap E)$. Thus, $p(E)=p(E-A)+p(A \cap E)=p^{\prime}(A-E)+$ $p^{\prime}(A \cap E)=p^{\prime}(A)=p(A)$, where the last equality holds by orbit-closedness of $\Gamma$.

Step 2: $p(F)=p\left(F^{\prime}\right)$ for all $p \in \Gamma$ and for all disjoint events $F, F^{\prime} \in \Sigma$ with $q(F)=q\left(F^{\prime}\right)=\beta<\alpha$.

Observe that $\beta<\frac{1}{2}$, so $\alpha-\beta<1-2 \beta$. Thus, by range-convexity of $q$, there exists $H \subseteq\left(F \cup F^{\prime}\right)^{c}$ with $q(H)=\alpha-\beta$. By Step 1 applied to sets $F \cup H$ and $F^{\prime} \cup H$, it follows that $p(F)+p(H)=p(F \cup H)=p(A)=$ $p\left(F^{\prime} \cup H\right)=p\left(F^{\prime}\right)+p(H)$; hence, $p(F)=p\left(F^{\prime}\right)$.

Step 3: $p(G)=q(G)$ for all $p \in \Gamma$ and for $G \in \Sigma$. 
Let $\gamma=q(G)$ and for each $n \in \mathbb{N}$ define $k_{n}=\sup \left\{k \mid \frac{k}{n} \leq \gamma\right\}$. Observe, that $\lim _{n \rightarrow \infty} \frac{k_{n}}{n}=\gamma$. For each $n \in \mathbb{N}$, by range-convexity of $q$, there exists a partition $\left\{F_{1}, \ldots, F_{n}\right\}$ of $F$ such that $q\left(F_{k}\right)=\frac{1}{n}$ for $k=1, \ldots, n$, sets $F_{1}, \ldots, F_{k_{n}} \subseteq G$, and sets $F_{k_{n}+2}, \ldots, F_{n} \subseteq G^{c}$. By Step 2, $p\left(F_{k}\right)=\frac{1}{n}$ for $k=1, \ldots n$, so $\frac{k_{n}}{n} \leq p(G) \leq \frac{k_{n}+1}{n}$. By letting $n$ to infinity, $p(G)=\gamma$.

Proof of Theorem 3. To see $(i) \Rightarrow(i i)$, observe that for any $r \in \mathbb{R}_{+}$let $C_{r}=\{p \in \Delta(S, \Sigma) \mid c(p)=r\}$ denote the level set of the cost function $c$. Observe that

$$
V(f)=\min _{p \in \Delta(S)} \int_{S}(u \circ f) \mathrm{d} p+c(p)=\min _{r \in \mathbb{R}_{+}} \min _{p \in C_{r}} \int_{S}(u \circ f) \mathrm{d} p+r .
$$

By weak monotone continuity and the proof of Corollary 4 in Sarin and Wakker [15] it follows that $\succsim$ is probabilistically sophisticated with respect to some $q \in \Delta^{\sigma}(S)$. By Theorem 14 of Maccheroni et al. [11], if $\succsim$ is probabilistically sophisticated with respect to $q \in \Delta^{\sigma}(S)$, then $c$ is rearrangement invariant, i.e., $p \sim_{c x} p^{\prime} \Rightarrow c(p)=c\left(p^{\prime}\right)$ for all $p, p^{\prime} \in \Delta(S)$. Thus, each $C_{r}$ is orbit-closed. Therefore, by Assumption 3 and Lemma 2, $C_{r}=\{q\}$ for all $r \in \mathbb{R}_{+}$. Thus,

$$
V(f)=\min _{r \in \mathbb{R}_{+}} \int_{S}(u \circ f) \mathrm{d} q+r=\int_{S}(u \circ f) \mathrm{d} q .
$$

The direction $(i i) \Rightarrow(i i i)$ is trivial. To see $(i i i) \Rightarrow(i)$, observe that by Theorem 2 Axiom A1 implies Assumption 2, which in turn implies Assumption 3.

\section{References}

[1] M. Allais. Le Comportement de l'Homme Rationnel devant le Risque: Critique des Postulats et Axiomes de l'Ecole Americaine. Econometrica, 21(4):503-546, 1953. 
[2] Simone Cerreia-Vioglio, Fabio Maccheroni, Massimo Marinacci, and Luigi Montrucchio. Uncertainty averse preferences. Working Paper, Universita Bocconi, 2008.

[3] Simone Cerreia-Vioglio, Fabio Maccheroni, Massimo Marinacci, and Luigi Montrucchio. Probabilistic sophistication, second order stochastic dominance, and uncertainty aversion. Working Paper, Universita Bocconi, 2009.

[4] S.H Chew and J.S. Sagi. Event Exchangeability: Probabilistic Sophistication Without Continuity or Monotonicity. Econometrica, 74(3):771$786,2006$.

[5] D. Ellsberg. Risk, Ambiguity, and the Savage Axioms. The Quarterly Journal of Economics, 75(4):643-669, 1961.

[6] I. Gilboa and D. Schmeidler. Maxmin expected utility with non-unique prior. Journal of Mathematical Economics, 18(2):141-153, 1989.

[7] S. Grant. Subjective Probability without Monotonicity: Or How Machina's Mom May Also Be Probabilistically Sophisticated. Econometrica, 63:159-159, 1995.

[8] Simon Grant and Atsushi Kajii. Probabilistically sophisticated multiple priors. Working Paper, Rice University, 2005.

[9] Lars Peter Hansen and Thomas J. Sargent. Robust control and model uncertainty. The American Economic Review, 91(2):60-66, 2001. ISSN 0002-8282.

[10] W. A. J. Luxemburg. Rearrangement Invariant Banach Function Spaces. Queens Papers in Pure and Applied Mathematics, 10:83-144, 1967. 
[11] Fabio Maccheroni, Massimo Marinacci, and Aldo Rustichini. Ambiguity aversion, robustness, and the variational representation of preferences. Econometrica, 74(6):1447 - 1498, 2006.

[12] Mark J. Machina and David Schmeidler. A more robust definition of subjective probability. Econometrica, 60(4):745-780, 1992.

[13] Massimo Marinacci. Probabilistic sophistication and multiple priors. Econometrica, 70(2):755-764, 2002.

[14] John Quiggin. A theory of anticipated utility. Journal of Economic Behavior and Organization, 3(4):323-343, 1982.

[15] Rakesh Sarin and Peter P. Wakker. Cumulative dominance and probabilistic sophistication. Mathematical Social Sciences, 40:191-196, 2000.

[16] C. Villegas. On qualitative probability $\sigma$-algebras. The Annals of Mathematical Statistics, 35(4):1787-1796, dec 1964.

[17] Menahem E. Yaari. The dual theory of choice under risk. Econometrica, 55(1):95-115, 1987. 